\title{
A Robust Suboptimal PD Servo Controller for an Oriented PV System
}

\section{Introduction}

In many practical situations parameters of servo systems are known as intervals only. This is determined by many factors: the system works outdoor in different weather conditions (summer/winter), parameters of moving parts during motion up or down are different, the system is strongly nonlinear, etc.

Problems of robust control for uncertain systems have been investigated by more Authors. As examples fundamental books of Dorato (2001 and later), Feintuch (1998), Jaulin (2001) or Grimble (2006) [2-5] can be pointed.

During control an uncertain-parameter system, a control performance, described by a suitable cost function, should be kept independently on strongly changing conditions. This job can be realised by properly tuned robust controller. Typically, in servo system a PD controller is applied and the "D" part is realized with the use of tachometric feedback.

In this paper the proposition of robust, suboptimal PD controller for servo system is proposed. The controller should be suboptimal in sense of cost function describing both an energy consumption during control and a settling time. Methods similar to presented in this paper were presented also in author's papers from years 2009-2011. The approach using robustness indices was presented for example in author's monograph from 2008. A new idea presented in this paper is the synthesis of robust PD controller realised with the use of tachometric feedback.

In paper the following problems were discussed:

- The considered control plant.

- Servo control system.

- The cost function and their minimization for plant with known and interval parameters.

- An example from area of PV systems.

* AGH University of Science and Technology, Krakow, Poland 


\section{Control plant under consideration}

The plant we deal with is described by the following elementary transfer function:

$$
G(s)=\frac{1}{T_{i} s(T s+1)}
$$

In (1) $T_{i}$ and $T$ denote uncertain parameters of the plant. They are described by positive interval numbers:

$$
\begin{aligned}
& T_{i}=\left[\underline{T_{i}} ; \overline{T_{i}}\right], \quad \underline{T_{i}}>0 \\
& T=[\underline{T} ; \bar{T}], \quad \underline{T}>0
\end{aligned}
$$

The approach presented here is based on the results presented by author for example in 2008 and 2009. The both parameters build the space of uncertain plant parameters $Q$ :

$$
Q=\left\{q \in I\left(R^{2}\right): q=\left[T_{i} ; T\right]^{T}\right\}
$$

The set (2) can be interpreted as a rectangle in $\boldsymbol{R}^{2}$ plane. Vertices of $Q$ are defined as follows:

$$
\begin{aligned}
& q_{l l}=\left[\underline{T_{i}} ; \underline{T}\right]^{\mathrm{T}} \\
& q_{l h}=\left[\underline{T_{i}} ; \bar{T}\right]^{\mathrm{T}} \\
& q_{h l}=\left[\bar{T}_{i} ; \underline{T}\right]^{\mathrm{T}} \\
& q_{h h}=\left[\overline{T_{i}} ; \bar{T}\right]^{\mathrm{T}}
\end{aligned}
$$

The uncertainty of the plant can be caused by many factors. For example, if the transfer function (1) represents an elevation drive of the oriented PV system, (working outdoor), the uncertainty is caused by extremely different weather conditions during the whole year, different conditions of work of drive during motion the moving part up and down and nonlinear dependences between elevation angle and input voltage signal given on the driving motor. 


\section{A servo control system for the considered plant}

The plant described above is controlled with the use of PD controller with "D" part realised as tachometric feedback. The scheme of the control system is shown in Figure 1.

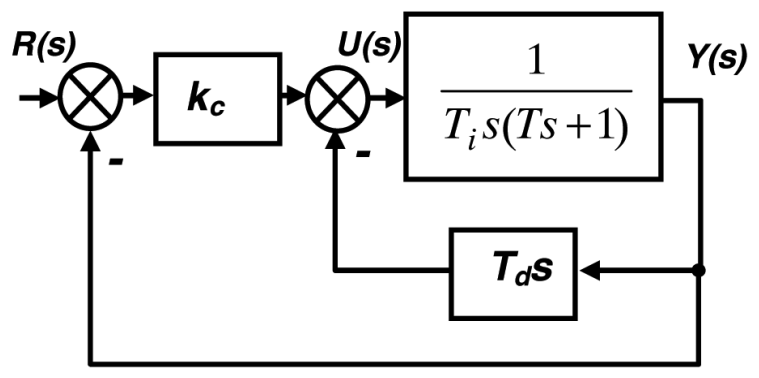

Fig. 1. The servo control system with tachometric feedback

In Figure $1 T_{d}$ denotes a differentiation time, $k_{c}$ denotes a proportional gain of controller. The both blocks realize a PD controller. Assume, that the reference value $r(t)$ is a unit step. This implies, that the Laplace transform of control signal $U(s)$ is equal:

$$
U(s)=\frac{k_{c} T_{i} T s^{2}+k_{c} T_{i} s}{T_{i} T s^{2}+\left(T_{i}+T_{d}\right) s+k_{c}} R(s)
$$

The denominator of (5) is a characteristic polynomial of the control system under consideration. It is equal:

$$
D_{c}(s)=T_{i} T s^{2}+\left(T_{i}+T_{d}\right) s+k_{c}
$$

During tuning a PD controller for the system we deal with the crucial problem is to achieve the shortest possible settling time without oscillations. If the parameters of plant are well known, then the PD controller meeting this assumption is simple to obtain and it has the following form:

$$
\begin{aligned}
k_{c} & \geq \frac{T_{i}}{4 T} \\
T_{d} & =2 \sqrt{T_{i} T k_{c}}-T_{i}
\end{aligned}
$$

The conditions (7) describe the situation, when the closed-loop system has one, dual, pure real root and can be obtained directly from condition describing the localisation of roots the polynomial (6). 
The situation turns to be more complicated, if the parameters of a plant are intervals, described by (2), (3) and (4). Then the condition in the simple, but strict form (7) is impossible to keep and it should be replaced by more "elastic" conditions, possible to meet for interval parameters of the plant:

$$
\begin{aligned}
& \forall\left[T_{i} ; T\right]=q \in I\left(R^{2}\right): \quad k_{c} \geq \max _{Q}\left(\frac{T_{i}}{4 T}\right) \\
& \forall\left[T_{i} ; T\right]=q \in I\left(R^{2}\right): T_{d} \geq \max _{Q}\left(2 \sqrt{T_{i} T k_{c}}-T_{i}\right)
\end{aligned}
$$

In (8) and (9) parameters $T_{i}$ and $T$ are positive intervals, described by (2). The conditions (8) and (9) guarantee, that the response of the control system will be always aperiodic, but the settling time of the closed-loop control system will be generally longer, than for system with known parameters and met strict condition (7). Possibilities of technical realization make us to formulate additional limitations for both parameters of controller:

$$
\begin{aligned}
& k_{c} \leq k_{c \max } \\
& T_{d} \leq T_{d \text { max }}
\end{aligned}
$$

Finally, the controller parameters $k_{c}$ and $T_{d}$ meeting (8), (9) and (10) build the space of permissible controller parameters $P_{s}$ :

$$
P_{S}=\left\{\begin{array}{l}
p_{s}=\left[k_{c} ; T_{d}\right]^{\mathrm{T}}: \max _{Q}\left(\frac{T_{i}}{4 T}\right) \leq k_{c} \leq k_{c \max }, \\
\max _{Q}\left(2 \sqrt{T_{i} T k_{c}}-T_{i}\right) \leq T_{d} \leq T_{d \max }, \\
{\left[T_{i} ; T\right]=q \in I\left(R^{2}\right)}
\end{array}\right\} \subset R^{2}
$$

The geometric interpretation of the set $P_{s}$ described by (11) is shown in Figure 2.

The low border of area $P_{S}$ shown in Figure 2 is described directly by (7). Additionally, remember, that the robust controller under consideration is dedicated to control an autonomic oriented PV system. This implies, that fundamental factors necessary to consider during control system construction are: an energy consumption and a settling time during control. This should be described by the suitable cost function. 


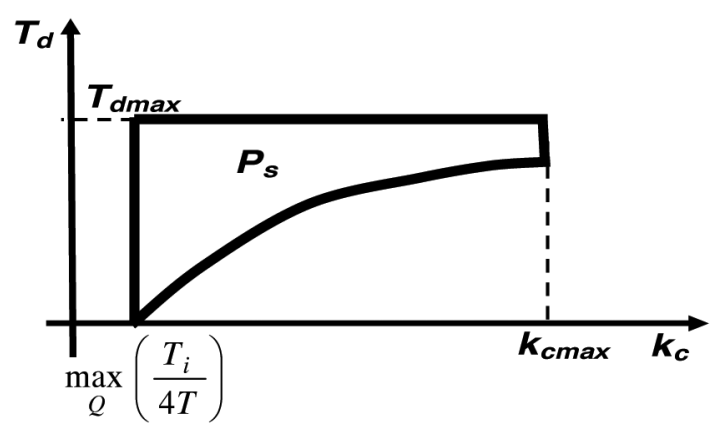

Fig. 2. The set of permissible parameters of a PD controller

\section{A cost function for the system under consideration}

\subsection{The system with known parameters}

From the above discussion it turns out, that the cost function for the discussed problem is required to describe both the energy consumption and settling time during control. The construction of minimal energetic controller for $2^{\text {nd }}$ order uncertain parameters plant was presented by Mitkowski and Oprzędkiewicz in [6-8].

The cost function construction and analysis will be started for the "energetic" factor. A factor describing the energy consumption during control will be denoted by $I_{e}$ and it can be expressed as underneath:

$$
I_{e}=\int_{0}^{\infty} u^{2}(t) d t
$$

The integral (12) can be presented in analytical form also (see for example [1]). This analytical form is obtained from equation (4), describing the Laplace transform of control signal in system. It has the following form:

$$
I_{e}=\frac{k_{c}^{2} T_{i} T+k_{c} T_{i}^{2}}{2 T_{i}+2 T_{d}}
$$

If we assume, that parameters of plant are exactly known and the derivative time of controller $T_{d}$ meets the strict condition (7), then the cost function (12) can be expressed as a function of proportional gain $k_{c}$ only:

$$
I_{e}=\frac{1}{4}\left(\sqrt{T_{i} T} k_{c}^{\frac{3}{2}}+\frac{T_{i}^{\frac{3}{2}}}{\sqrt{T}} k_{c}^{\frac{1}{2}}\right)
$$


Furthermore assume, that the parameters of controller are localised directly in vertex $P_{l l}$ of set $P_{S}$ (see Fig. 2). They are described as follows:

$$
\begin{aligned}
k_{c} & =\frac{T_{i}}{4 T} \\
T_{d} & =0
\end{aligned}
$$

The parameters (15) describe the "pure" proportional controller and the cost function (14) is determined by plant parameters only. It is equal:

$$
I_{e v}=\frac{5 T_{i}^{2}}{32 T}
$$

We can notice at once, that the minimum of cost function $I_{e}$ described by (14) in the area $P_{S}$ is met in vertex $P_{l l}$, when the controller is described by (15) and the minimal value of cost function is equal (16). The controller described by (15) guarantees the minimal energy consumption during control, but the settling time of closed-loop control system is too long to accept. Unfortunately, the requirement of shorting settling time is in collision with requirement of minimal energy consumption, because the energy consumption decreases with shorting the settling time. This can be noticed directly with the use of equation (14), which describes the situation with the minimal settling time.

The analytical factor describing the settling time as a function of plant and controller parameters can have different forms. The simple idea is to describe it with the use of dumping coefficient of the closed-loop system. The dumping coefficient is determined by the root of the characteristic polynomial localised closely to zero. Let us denote it by $\alpha_{c}$. It can be generally described as underneath:

$$
\alpha_{c}\left(p_{s}, q\right)=\min _{i=1,2}\left|\operatorname{Re}\left(\lambda_{i}\left(p_{s}, q\right)\right)\right|
$$

where $\lambda_{1}\left(p_{s}, q\right)$ and $\lambda_{2}\left(p_{s}, q\right)$ denote roots of the characteristic polynomial of system, described by (6). The bigger value of the coefficient (17) implies the smaller value of the settling time in the system. This implies, that the cost function, describing the settling time, can be proposed in the following form:

$$
I_{t}=\frac{1}{\alpha_{c}\left(p_{s}, q\right)}
$$

Consequently the cost function, describing both the energy consumption and the settling time can be defined as the weighted sum of factors (12) and (18):

$$
I\left(p_{s}, q\right)=w_{1} I_{e}\left(p_{s}, q\right)+w_{2} I_{t}\left(p_{s}, q\right)
$$


In (19) $w_{1}$ and $w_{2}$ denote the normalized weight coefficients. If we assume, that the strict condition (7) is met, then the dumping coefficient (17) turns to the following simple form:

$$
\alpha_{c}=\sqrt{T_{i} T} k_{c}^{-\frac{1}{2}}
$$

and consequently, the cost function (18) turns to the following form:

$$
I_{t}=\sqrt{T_{i} T} k_{c}^{-\frac{1}{2}}
$$

Finally, the cost function (19) with respect to (7), (8), (14) and (21) turns to the following form:

$$
I\left(p_{s}, q\right)=\frac{1}{4} w_{1} \sqrt{T_{i} T} k_{c}^{\frac{3}{2}}+\frac{1}{4} w_{1} \frac{T_{i}^{\frac{3}{2}}}{\sqrt{T}} k_{c}^{\frac{1}{2}}+w_{2} \sqrt{T_{i} T} k_{c}^{-\frac{1}{2}}
$$

The gain $k_{c}$ minimizing the cost function (22) can be analytically calculated. It can be expressed as follows:

$$
k_{c}^{0}=\frac{\sqrt{\frac{1}{16} w_{1}^{2} \frac{T_{i}^{2}}{T}+\frac{2}{3} w_{1} w_{2} T}-\frac{1}{4} w_{1} \frac{T_{i}}{\sqrt{T}}}{\frac{2}{3} w_{1} \sqrt{T}}
$$

The optimal gain $k_{c}^{0}$, described by (23) must meet the condition (8), assuring the positive value of $T_{d}$ parameter. If the condition (8) is not met, then it can be easily seen, that the minimum of (22) is reached in vertex $q_{l l}$ of set $Q$.

The optimal value of the differentiation time $T_{d}^{0}$ can be calculated with the use of (7) and (23):

$$
T_{d}^{0}=2 \sqrt{T_{i} T k_{c}^{0}}-T_{i}
$$

Consequently, the optimal PD controller is expressed as the following vector $p_{s}^{0} \in P_{s}$ :

$$
p_{s}^{0}=\left[k_{c}^{0} ; T_{d}^{0}\right]^{T} \in P_{s}
$$

Finally, the minimal value of cost function for $p_{s}^{0}$ can be calculated with the use of (22) and (23):

$$
I^{0}\left(p_{s}, q\right)=I\left(p_{s}^{0}, q\right)
$$


The construction of the optimal PD controller presented above is simple for plant with known parameters, but for an interval parameter plant the optimization problem presented above turns to be more complicated. This implies, that the problem formulation presented above before further analysis should be re-defined with respect to uncertainty the plant. This will be presented in the next section.

\subsection{The system with interval parameters}

The optimal PD controller described by (23) and (24) can be uniquely assigned for one, fixed value of vector $q$ only and it will not be optimal for another values of $q$. If the interval conditions (8) and (9) are met, then the closed-loop control system will be aperiodic, but not optimal in the sense of the cost function (19), because the strict condition (7) is impossible to keep for interval numbers.

The analysis of the interval case requires us to express the both factors of cost function (19) by their general forms, because the simple, but strict condition (14) and (21) are not true for interval parameters.

The factor associated to the energy consumption is described by the general expression (13), the factor associated to the settling time has the general form (18), the dumping coefficient is expressed by (17), but the roots of the characteristic polynomial (6) have the following general form:

$$
\lambda_{1,2}=\frac{-\left(T_{i}+k_{c} T_{d}\right) \mp \sqrt{\left(T_{i}+k_{c} T_{d}\right)^{2}-4 T_{i} T k_{c}}}{2 T_{i} T}
$$

During further analysis a geometrical approach proposed by author will be applied. Notice, that for the fixed vector $p_{s}$ and the whole set $Q$ the cost function (19) can be interpreted as a surface $V_{I}$ in $\boldsymbol{R}^{3}$ space. This surface can be defined as follows:

$$
V_{I}=\left\{\begin{array}{l}
v\left(p_{s}^{0}, q\right) \in R^{3}: v\left(p_{s}^{0}, q\right)=I\left(p_{s}^{0}, q\right), \\
p_{s}^{0}=\left[k_{c}^{0}, T_{d}^{0}\right]^{\mathrm{T}}, q \in Q
\end{array}\right\}
$$

We are not able to construct a controller, which would be optimal for the whole surface $V_{I}$. To propose a controller we need to "replace" the whole surface by one, selected point $V^{*}$ in $V_{I}$. Denote coordinates of point $V^{*}$ by $q^{*}$. The controller is constructed with the use of equations (23), (24) and vector $q^{*}$. Denote this controller by $p_{s}{ }^{*}$. Notice, that the controller $p_{s}{ }^{*}$ is optimal in sense cost function (19) in point $V^{*}$ and it is not optimal for the rest of set $Q$, but it is expected, that this controller $p_{s}{ }^{*}$ should be sub-optimal in the rest of set $Q$ and meet the assumptions (8) and (9) in the whole set $Q$ (these assumptions guarantee non oscillatory response of the system in the whole set $Q$ ). An idea of "sub-optimality" requires to intro- 
duce a formal measure of it. This measure should be expressed by the suitable cost function, based onto the cost function for system with known parameters. Denote this "suboptimal"cost function by $I^{*}$.

The first idea is to select the suboptimal cost function $I^{*}$ as the "worst case": we select as $I^{*}$ such a function $I$ (19) for which it's value calculated with the use of (23), (24) and (26) is maximal inside the area $Q$. Denote this function by $I^{*}{ }_{w}$. It can be expressed as follows:

$$
I_{w}^{*}=\max _{Q} I\left(p_{s}, q_{w}\right)
$$

In (29) $q_{w}$ denotes the vector $q$, for which the maximum of cost function is reached . It is easy to see, that this maximum is localised in vertex $q_{h h}$ of set $Q$, defined by (4):

$$
q_{w}=q_{h h}
$$

Denote the suboptimal controller built with the use of (30) by $p_{s w}^{*}$. It is built with the use of (23), (24) and vector of uncertain parameters (30). An alternative idea proposed by author is to assign the function $I^{*}$ as a "centre of gravity" the surface $V_{I}$ : The centre of gravity of the surface $V_{I}$ is localised in point $q_{c o g}$ :

$$
q_{\operatorname{cog}}=\left[T_{i \operatorname{cog}} ; T_{\operatorname{cog}}\right]^{\mathrm{T}}
$$

where:

$$
\begin{gathered}
T_{i \operatorname{cog}}=\frac{\int T_{i} V_{I} d T_{i}}{\int V_{I} d Q} \\
T_{\operatorname{cog}}=\frac{\int T V_{I} d T}{\int V_{I} d Q}
\end{gathered}
$$

Denote the cost function calculated with the use of (32) by $I_{c o g}^{*}$. It can be expressed as follows:

$$
I_{c o g}^{*}=I\left(p_{s}, q_{c o g}\right)
$$

Consequently, denote the suboptimal controller built with the use of (33) by $p_{\text {scog. }}^{*}$. It is built with the use of (23), (24) and vector of uncertain parameters (31)-(32).

The above approach was applied to construct the optimal LQ controller for second order interval plant (see [6-8]). The synthesis of the suboptimal controller with the use of one of functions (29) or (33) can be easily run with the use of numerical methods. This will be shown in the example. 


\section{An Example}

As an example consider the construction of suboptimal PD controller for an interval plant described by the following interval parameters (describing a real plant):

$$
\begin{aligned}
T_{i} & =[1.8 ; 2.3][\mathrm{s}] \\
T & =[0.5 ; 0.7][\mathrm{s}]
\end{aligned}
$$

The parameters (34) describe the elevation system for an experimental PV system localised in AGH University of Science and Technology, Dept. of Automatics and Biomedical Engineering. An input of the system is a required elevation angle, an output is a real elevation angle. The control signal is an input voltage given on DC motor. Assume that the maximal values of PD controller parameters are equal:

$$
\begin{aligned}
& k_{c \text { max }}=5 \\
& T_{d \text { max }}=5[\mathrm{~s}]
\end{aligned}
$$

Furthermore, estimate the lower border of controller parameters, described by (8). In our example they are equal:

$$
k_{c} \geq 1.15
$$

During synthesis the controller the weight coefficients $w_{1}$ and $w_{2}$ were equal:

$$
\begin{aligned}
& w_{1}=0.25 \\
& w_{2}=0.75
\end{aligned}
$$

The above weights allow us to build controllers more dedicated to assure short settling time, than low energy consumption.

The surface $V_{I}$ described by (28) for example is shown in Figure 3.

For our example, the maximum of the cost function is localised in vertex $q_{h l}$. The centre of gravity of the surface $V_{I}$ is localised in point $q_{c o g}$. The coordinates of both points, parameters of the both the PD controllers and the values of cost function are shown in Table 1.

Table 1 shows, that the "worst case" controller can be constructed for border case only, because the minimum of cost function (22) for our example is localised outside the set $P_{s}$. 


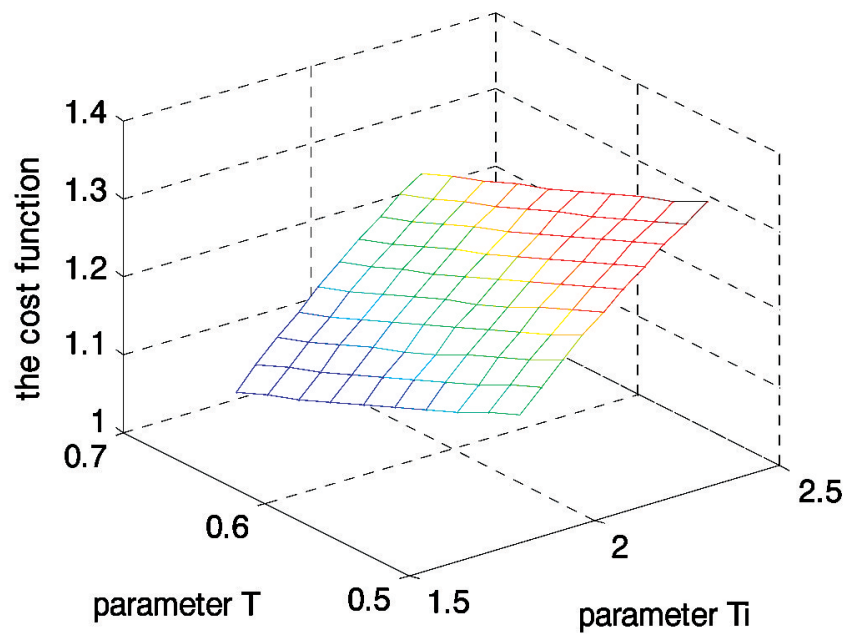

Fig. 3. The surface $V_{I}$ for example

Table 1

Suboptimal PD controllers

\begin{tabular}{|c|c|c|c|}
\hline $\begin{array}{c}\text { Point } \\
\text { of set } Q\end{array}$ & Values & $\begin{array}{c}\text { Version of PD } \\
\text { controller }\end{array}$ & $\begin{array}{c}\text { Value of cost } \\
\text { function }(22)\end{array}$ \\
\hline \multirow{2}{*}{$q_{w}$} & $\begin{array}{c}T_{i}=2.3[\mathrm{~s}] \\
T=0.5[\mathrm{~s}]\end{array}$ & $\begin{array}{c}p_{s w}{ }^{*}: \\
k_{c}^{0}=1.15 \\
T_{d}^{0}=0.0[\mathrm{~s}]\end{array}$ & 1.1633 \\
\hline \multirow{2}{*}{$q_{\operatorname{cog}}$} & $\begin{array}{c}T_{i}=2.0443[\mathrm{~s}] \\
T=0.5977[\mathrm{~s}]\end{array}$ & $\begin{array}{c}p_{\text {scog }}{ }^{*}: \\
k_{c}{ }^{0}=1.1963 \\
T_{d}^{0}=0.3738[\mathrm{~s}]\end{array}$ & 1.1068 \\
\hline
\end{tabular}

To compare properties of control systems built with the use of the both presented methods of the simulations with the use of MATLAB/SIMULINK were run. During simulations the step responses of the control system for both the proposed controllers and four vertex plants described by (4) and (34) were assigned. The time diagrams of all responses are shown in Figures 4 and 5.

From the both diagrams we can conclude at once, that the "worst case" controller assures shorter settling time, but the response for vertex $q_{l h}$ has an overshoot, which is not permissible. The "centre of gravity" controller assures a little bit longer settling time, but all responses do not have overshoot.

Both the controllers assure the comparable settling time in range between 3-5 [s] for moving the plant from position " 0 " to " 1 " and this value can be interpreted as absolutely sufficient in the considered application. 


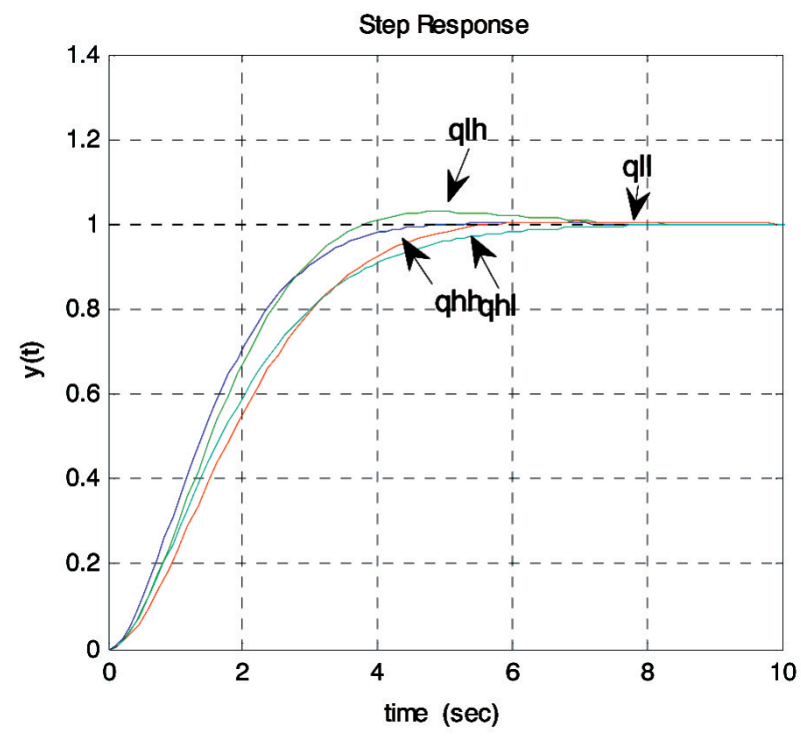

Fig. 4. The step responses of the servo control system and controller $p_{s w}$

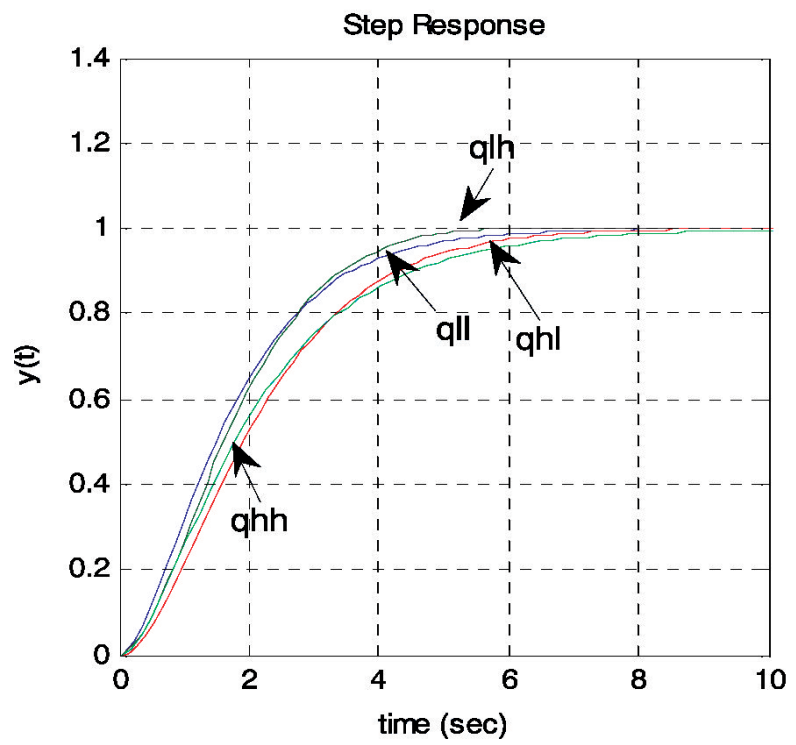

Fig. 5. The step responses of the servo control system and controller $p_{\text {scog }}$

\section{Acknowledgements}

This paper is sponsored by the NCN National Science Centre grant 6693/B/T02/2011/40. 


\section{References}

[1] Byrski W., Obserwacja i sterowanie w systemach dynamicznych [Control and observation in dynamic systems]. Uczelniane Wydawnictwa Naukowo-Dydaktyczne, Kraków, 2007 (in Polish).

[2] Dorato P., Fortuna L., Muscato G., Robust control for unstructured perturbations-an introdution. Berlin, Springer, 1992.

[3] Feintuch A., Robust control theory in Hilbert space. New York, Springer, 1998.

[4] Grimble M., Robust industrial control systems: optimal design approach for polynomial systems. Chichester, J. Wiley \& Sons, 2006.

[5] Jaulin L., Kieffer M., Didrit O. Walter E., Applied interval analysis. Springer, 2001.

[6] Mitkowski W., Oprzędkiewicz K., A discrete minimal energy control for 2'nd order uncertainparameter plant. XVII State Automatics Conference, KKA'2011: Kielce-Cedzyna, 1922.06.2011, 545-556 (in Polish).

[7] Mitkowski W., Oprzędkiewicz K., Optimal control for an uncertain parameters, 2'nd order plant with the use of discrete controller. 25th IFIP TC 7 Conference on „System modeling and optimization": Berlin, September 12-16, 2011: book of abstracts.

[8] Mitkowski W., Oprzędkiewicz K., A minimum-energy controller for 2nd order uncertain parameter plant. Automatyka (semi-annual AGH-UST), t. 15, z. 2, 2011, 355-363 (in Polish).

[9] Oprzędkiewicz K., Robust PID controller for a class of linear uncertain parameter systems. Electrical Review, R. 85, no 3, 2009, 22-27 (in Polish).

[10] Oprzędkiewicz K., Practical control of dynamic systems with point spectrum and interval parameters. Monograph edited by AGH UST, no 186, 2008 (in Polish). 